\section{Adlayers based on molecular platforms of trioxatriangulenium $\dagger$}

\author{
Sonja Kuhn, ${ }^{a}$ Ulrich Jung, ${ }^{a}$ Sandra Ulrich, ${ }^{b}$ Rainer Herges ${ }^{* b}$ and Olaf Magnussen $* a$ \\ Received 3rd May 2011, Accepted 21st June 2011 \\ DOI: $10.1039 / \mathrm{c} 1 \mathrm{cc12598b}$
}

The platform approach for preparation of molecular adlayers with freestanding functional groups was extended to systems on the basis of the trioxatriangulenium ion. Self-assembled monolayers of these compounds were prepared on $\mathrm{Au}(111)$ surfaces and characterized by scanning tunneling microscopy, revealing highly-ordered structures.

Organic surface functionalization with defined vertical architecture is of considerable current interest. ${ }^{1}$ We recently introduced the platform approach for preparation of wellordered adlayers with freestanding functional groups on metal surfaces and illustrated its feasibility by employing derivatives of the triazatriangulenium (TATA) ion. $^{2-5}$ The triangularshaped TATA platform can be functionalized either vertically at the central carbon or laterally at the outer nitrogen atoms and thus serves as a chemically very modular and versatile template. Up to now, more than 30 TATA derivatives with different functional groups have been synthesized. ${ }^{4}$ Adlayers of these compounds have been studied by STM, ${ }^{2,3,5}$ different spectroscopies, ${ }^{5}$ and electrochemical methods. ${ }^{5,3}$ Using these techniques the TATA derivatives were shown to form hexagonally-ordered adlayers where the platforms adsorb flat on the metal substrate, the intermolecular distances can be varied by the attached side groups, and the central functional groups are oriented perpendicularly to the surface. Although this approach turned out to be an excellent method for preparation of highly-ordered functional adlayers, the employed TATA platforms have some disadvantages. First, it is not possible to prepare functional TATA adlayers by vacuum deposition, because they decompose below their sublimation point. This limits their applicability for studies under ultrahigh vacuum conditions. Second, the inevitably present side groups attached to the nitrogen atoms partially reside above the TATA layer and (for the employed alkane chains) do not seem to exhibit well-defined positions, which reduces the structural order in the adlayers.

\footnotetext{
${ }^{a}$ Institut für Experimentelle und Angewandte Physik, Christian-Albrechts-Universität zu Kiel, Leibnizstraße 19, 24118 Kiel, Germany.E-mail: magnussen@physik.uni-kiel.de; Fax: + 49431880 4884; Tel: + 494318805579

${ }^{b}$ Otto-Diels-Institut für Organische Chemie, Christian-Albrechts-Universität zu Kiel, Otto-Hahn-Platz 4, 24098 Kiel, Germany.E-mail: rherges@oc.uni-kiel.de; Fax: + 49431880 1558; Tel: + 494318802440

$\dagger$ This article is part of the ChemComm 'Molecule-based surface chemistry' web themed issue.
}

In this work we use an alternative molecular platform system, derivatives of the trioxatriangulenium (TOTA) ion (Fig. 1), which does not have these disadvantages. TOTA is structurally closely related to TATA, but exhibits three oxygen atoms in the platform instead of nitrogen atoms with attached side groups and thus a significantly reduced molecular mass. Similarly as for TATA perpendicular as well as lateral functionalization is possible, although the latter only at para or meta positions of the benzene rings in the platform. The bond strength of vertically attached functional groups to the central carbon atom of the TOTA platform should be increased as compared to TATA, making these molecules better suited for adlayer preparation via vacuum deposition. The TOTA derivatives have been extensively studied since the $1960 \mathrm{~s},{ }^{6-8}$ but up to now not in context of surface functionalization. As we will show in the following STM studies, highly-ordered adlayers of TOTA and functional TOTA derivatives can be prepared on $\mathrm{Au}(111)$ surfaces, demonstrating the feasibility to extend the platform concept to this new class of molecules.

In the experiments, the pure TOTA platform, ${ }^{6-8}$ a TOTA derivative with diethylamine side groups $\left(\mathrm{DEA}_{3}-\mathrm{TOTA}^{8}\right)$, and a vertically methyl-functionalized TOTA $\left({\left.\mathrm{Me}-\mathrm{TOTA}^{7}\right)}^{7}\right.$ were employed (Fig. 1), which were synthesized as described in the literature. The ionic species (TOTA and DEA DOTA) were $_{3}$ synthesized as $\mathrm{BF}_{4}{ }^{-}$salts. All compounds were characterized by NMR and mass spectroscopy and have a purity of $>99 \%$. The vertically functionalized Me-TOTA was found to be stable upon sublimation at $88{ }^{\circ} \mathrm{C}$, as confirmed by NMR spectroscopy. Adlayers of these compounds were prepared on single-crystalline $\mathrm{Au}(111)$ substrates, cleaned by flame annealing, by 30 to $60 \mathrm{~min}$ immersion into solutions of the molecules in ethanol or dichloromethane and subsequent rinsing with the solvent. STM studies were performed under ambient conditions with a PicoPlus STM and Pt/Ir tips. The measurements were carried out in constant current mode at tunneling currents of 30 to $80 \mathrm{pA}$ and bias voltages of 200 to $400 \mathrm{mV}$. Lateral drift in the STM images was corrected with a dedicated software.

STM images of TOTA (Fig. 2a) and Me-TOTA adlayers (Fig. $2 \mathrm{~b}$ and $\mathrm{c} 2$ ), prepared by immersion in a $1 \mathrm{mM}$ solution of

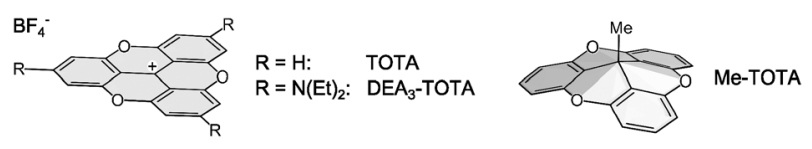

Fig. 1 TOTA derivatives used for preparation of adlayers on $\mathrm{Au}(111)$. 


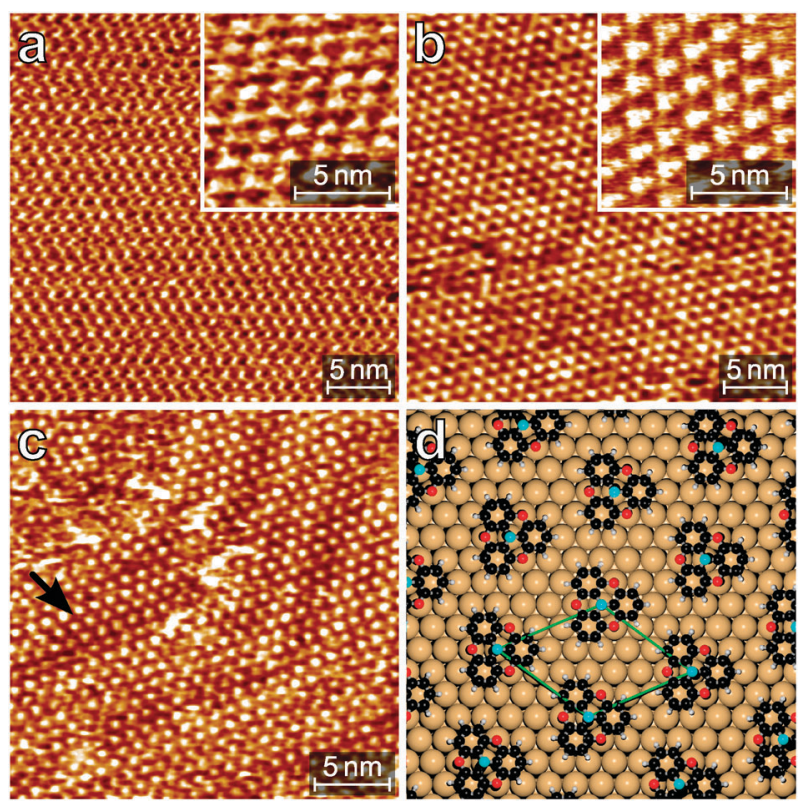

Fig. 2 STM images of (a) TOTA and (b,c) Me-TOTA adlayers on $\mathrm{Au}(111)$. (d) Structural model of these adlayers.

the compounds in dichloromethane at room temperature, show simple hexagonal structures with lattice constants of $11.9 \pm 0.5 \AA$ and $12.8 \pm 0.2 \AA$, respectively. Two rotational domains are observed. These structural parameters are in good agreement with a commensurate $(\sqrt{19} \times \sqrt{19}) R 23.4^{\circ}$ superstructure (see Fig. 2d), as also previously identified for Oct $_{3}$-TATA adlayers. ${ }^{2,3,5}$ High-resolution STM images (Fig. 2a and $b$, inset) reveal the triangular shape of the molecules, indicating a planar adsorption of the bare and vertically functionalized platforms and a uniform orientation of the molecules. As in our previous studies of TATA adlayers, ${ }^{3,5}$ no indications for the $\mathrm{BF}_{4}{ }^{-}$counter ions were found. Most probably, these are not coadsorbed on the surface, which can be explained by charge transfer from the substrate to the adsorbate molecules. The overall structural quality of the adlayers is very high, with typical domain sizes of $>100 \mathrm{~nm}$ and a low density of point defects, exceeding even that of TATA adlayers. ${ }^{3}$ This suggests a higher surface mobility of TOTA as compared to TATA platforms, which may be explained by a lower adsorption energy expected for the former species.

Whereas the lateral structures of TOTA and Me-TOTA seem to be identical, a notable difference is found in their interaction with the Au substrate: in the presence of the TOTA adlayer the $\mathrm{Au}(111)$ surface does not exhibit the "herringbone" reconstruction, indicating that the latter is lifted by the molecule-substrate interactions. In contrast, large "herringbone" reconstructed areas can be observed in STM images of $\mathrm{Au}(111)$ covered by Me-TOTA adlayers, as illustrated in Fig. 2c, where the characteristic double stripe pattern associated with the $(22 \times \sqrt{3})$ unit cell of this surface reconstruction ${ }^{9}$ is superimposed on the smaller hexagonal adlayer structure. The weaker Me-TOTA surface interaction suggested by these observations may potentially originate in the less planar shape of this functionalized molecule as compared to TOTA or the different charge state. Otherwise, the morphology of the Au substrate is only marginally affected by the formation of these adlayers, similarly as for TATA adlayers. ${ }^{3}$

A surprising result of these studies is the relatively low surface density of the TOTA and Me-TOTA adlayers of 0.053 monolayers (ML). In particular, this is significantly lower than that of $\operatorname{Pr}_{3}$-TATA adlayers, which form a $(\sqrt{13} \times \sqrt{13}) R 13.9^{\circ}$ superstructure with a coverage of $0.077 \mathrm{ML},{ }^{3}$ although both the bare TATA and TOTA platforms exhibit virtually identical dimensions. Obviously, the molecular arrangement is not solely determined by packing constraints, but by more complex effects, involving interactions with the Au substrate. The simple commensurate superstructure found for these adlayers implies a well-defined adsorption geometry, where the platform molecules occupy specific sites of the substrate. Support for this comes from a detailed inspection of the adlayer structure on reconstructed surface areas. Here, the in-plane modulation of the positions of the Au surface atoms, which change within the $(22 \times \sqrt{3})$ unit cell from fcc via bridge to hep sites relative to the underlying bulk lattice, ${ }^{9}$ is mimicked by an analogous lateral modulation within the adlayer (see the row indicated by an arrow in Fig. 2c). This provides clear evidence for a highly site-specific adsorption of the TOTA platform. Whether this is caused by an influence of the adsorption geometry on the dispersive interactions (e.g. a site-dependent variation of the molecule-surface spacing) or by local bonding (e.g. involving the $\mathrm{O}$ atoms of the platforms) is currently not clear, however.

Also for the laterally functionalized DEA $_{3}$-TOTA molecules self-assembly of adlayers from solution was possible under the same conditions. However, preparing adlayers of these molecules by the same method as that of the TOTA and Me-TOTA adlayers resulted in poorly ordered structures, exhibiting only
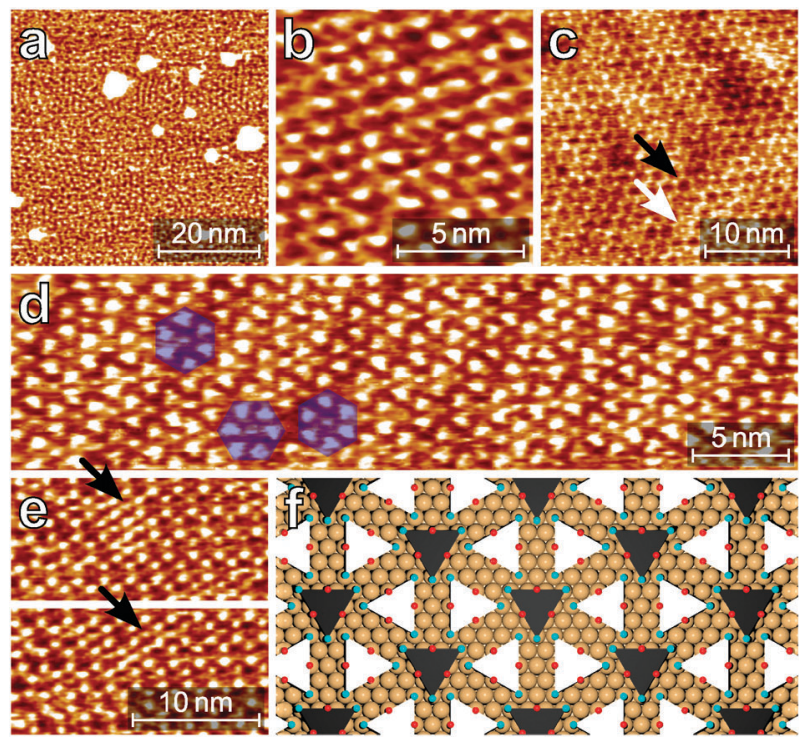

Fig. 3 STM images of $\mathrm{DEA}_{3}$-TOTA adlayers on $\mathrm{Au}(111)$, showing the (a) disordered adlayer, (b) the hexagonal adlattice, and (c) the "honeycomb" superstructure. High-resolution images showing (d) the molecular orientations and (e) dynamic fluctuations in the adlayer. (f) Model of the "honeycomb" structure. 
local order or small hexagonally-ordered domains (Fig. 3a). In contrast, for samples prepared from diluted solution at elevated temperatures $\left(10 \mu \mathrm{M}\right.$ solution in ethanol at $\left.60^{\circ}\right)$, highly-ordered adlayers are found. The predominant structural motive is a hexagonal lattice with an intermolecular spacing of $11.3 \pm 0.3 \AA$ (Fig. 3b). Together with the absence of different rotational domains and the parallel orientation of this adlattice to the Au substrate lattice (determined by comparison with the underlying $\mathrm{Au}$ reconstruction), this spacing is in excellent agreement with a $(4 \times 4)$ adsorbate structure. Surprisingly, the corresponding coverage of $0.063 \mathrm{ML}$ is slightly higher than that of the smaller TOTA platform. Again this may be caused by the combined influence of the highly site-specific bonding of these molecules and steric effects. The "herringbone" reconstruction of the $\mathrm{Au}$ substrate beneath the $\mathrm{DEA}_{3}$-TOTA adlayers is almost perfectly preserved, indicating that the interactions between the substrate and adsorbate molecules are weaker than in adlayers of the two other compounds.

In the majority of the STM images a more complex structural arrangement is observed, however (Fig. 3c). Here every third platform molecule of the $(4 \times 4)$ lattice appears less pronounced (Fig. 3c, white arrow) or to be missing completely (Fig. 3c, black arrow). The remaining molecules form a well defined "honeycomb" superstructure, which has a lattice constant of $19.7 \pm 0.4 \AA$ and corresponds to a $(\sqrt{48} \times \sqrt{48}) R 30^{\circ}$ structure relative to the $\mathrm{Au}$ substrate lattice. According to high-resolution images the six molecules of each "honeycomb" are either oriented with one corner to the center or all rotated by a distinct angle, resulting in an arrangement where the edges of the triangular shaped molecules are roughly parallel (examples marked by blue hexagons in Fig. 3d). The different molecular rotations seem to coexist without clear phase separation and may change dynamically under ambient conditions. Independent of the rotation, all molecules in the "honeycomb" network can be placed with the $\mathrm{O}$ and $\mathrm{N}$ atoms of the $\mathrm{DEA}_{3}$-TOTA platform occupying identical sites on the $\mathrm{Au}(111)$ substrate. As an example, this is shown for the case of unrotated molecules in Fig. 3f. In contrast, molecules in the center of the "honeycombs" have to reside on different, apparently less favorable sites. Additionally, occupation of these positions may be impeded by steric hindrances, caused by the attached ethyl groups. Both of these effects may lead to the apparently lower stability of platform molecules in these positions, manifesting in the lower occupancy of the "honeycomb" centers. Furthermore, pronounced dynamic fluctuations of the molecules in these sites are observed, as e.g. visible in the two subsequently recorded images in Fig. 3e, where molecules can be seen to hop to neighboring "honeycomb" centers (example marked by arrows). The less pronounced appearance and the observation of sudden changes (within one scan line) of platforms in "honeycomb" centers likewise may be explained by a high mobility of these molecules. The total occupancy of these centers seems to vary locally and with sample preparation, leading to coverages in between that of the pure "honeycomb" network (0.042 ML) and of the full $(4 \times 4)$ structure. Hence, these data may be rationalized by a dynamic molecular network with two molecular sites of different stability.

In summary, we have shown that derivatives of the trioxatriangulenium ion form highly ordered adlayers on $\mathrm{Au}(111)$ surfaces. This extension of the platform concept demonstrates its wide-ranged applicability and opens up new perspectives for the preparation of such functional adlayers.

We gratefully acknowledge financial support by Deutsche Forschungsgemeinschaft via Sonderforschungsbereich 677.

\section{Notes and references}

1 C. Wöll, Angew. Chem., Int. Ed., 2009, 48, 8406-8408.

2 B. Baisch, D. Raffa, U. Jung, O. Magnussen, C. Nicolas, J. Lacour, J. Kubitschke and R. Herges, J. Am. Chem. Soc., 2009, 131, 442-443.

3 S. Kuhn, B. Baisch, U. Jung, T. Johannsen, J. Kubitschke, R. Herges and O. Magnussen, Phys. Chem. Chem. Phys., 2010, 12, 4481-4487.

4 J. Kubitschke, C. Näther and R. Herges, Eur. J. Org. Chem., 2010, 5041-5055.

5 U. Jung, S. Kuhn, U. Cornelissen, F. Tuczek, T. Strunskus, V. Zaporojtchenko, J. Kubitschke, R. Herges and O. Magnussen, Langmuir, 2011, 27, 5899-5908.

6 J. C. Martin and R. G. Smith, J. Am. Chem. Soc., 1964, 86, 2252-2256.

7 M. Lofthagen, R. VernonClark, K. K. Baldridge and J. S. Siegel, J. Org. Chem., 1992, 57, 61-69.

8 B. W. Laursen, F. C. Krebs, M. F. Nielsen, K. Bechgaard, J. B. Christensen and N. Harrit, J. Am. Chem. Soc., 1998, 120, 12255-12263.

9 J. V. Barth, H. Brune, G. Ertl and R. J. Behm, Phys. Rev. B: Condens. Matter, 1990, 42, 9307-9318. 